\title{
Nanogel-based nasal ghrelin vaccine prevents obesity
}

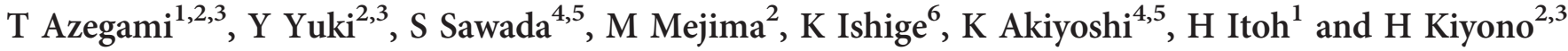

Obesity is associated with multiple comorbidities such as cardiovascular diseases and has a huge economic impact on the health-care system. However, the treatment of obesity remains insufficient in terms of efficacy, tolerability, and safety. Here we created a nasal vaccine against obesity for the first time. To avoid the injectable administration-caused pain and skin-related adverse event, we focused on the intranasal route of antigen delivery. We developed a vaccine antigen (ghrelin-PspA (pneumococcal surface protein A)), which is a recombinant fusion protein incorporating ghrelin, a hormone that stimulates food intake and decreases energy expenditure, and PspA, a candidate of pneumococcal vaccine as a carrier protein. Ghrelin-PspA antigen was mixed with cyclic di-GMP adjuvant to enhance the immunogenicity and incorporated within a nanometer-sized hydrogel for the effective antigen delivery. Intranasal immunization with ghrelin-PspA vaccine elicited serum immunoglobulin $G$ antibodies against ghrelin and attenuated body weight gain in diet-induced obesity mice. This obesity-attenuating effect was caused by a decrease in fat accumulation and an increase in energy expenditure that was partially due to an increase in the expression of mitochondrial uncoupling protein 1 in brown adipose tissue. The development of this nasal vaccine provides a new strategy for the prevention and treatment of obesity.

\section{INTRODUCTION}

Obesity is an independent risk factor for cardiovascular diseases. ${ }^{1}$ The prevalence of obesity is increasing, and 2.1 billion people worldwide were classed as being obese in $2013 .^{2}$ Obesity is predicted to cost the US health-care system $\$ 48-\$ 66$ billion per year by $2030 .{ }^{3}$ Despite the burden of obesity on the health-care system and economy, the treatments available for obesity are far from satisfactory in terms of efficacy, tolerability, and safety. Clinical trials have shown that not only clinically available antiobesity drugs reduce body weight in humans but also that the loss from baseline body weight is $<10 \%$ when compared with placebo-treated groups. ${ }^{4-8}$ In addition to their marginal effects on weight reduction, these antiobesity drugs are associated with controversial adverse effects, such as steatorrhea, malabsorption of liposoluble vitamins, and liver injury in case of orlistat; ${ }^{9-11}$ and carcinogenicity, psychiatric events, and valvulopathy in case of lorcaserin. ${ }^{11}$ Therefore, more effective and safer strategies for the treatment of obesity are required.

Among the numerous endogenous hormones, ghrelin is the only known circulating peripheral hormone that promotes body weight gain by stimulating food intake and decreasing energy expenditure. ${ }^{12}$ Ghrelin is an endogenous peptide comprising 28 amino acids and a ligand for growth hormone secretagogue receptor (GHSR) ${ }^{13}$ Ghrelin is not only mainly produced by X/A-like endocrine cells in the stomach ${ }^{14}$ but is also ubiquitously expressed in the intestine, pancreas, and pituitary gland. ${ }^{15}$ n-Octanoylation (acylation) at serine 3 residue, which is mediated by the enzyme ghrelin O-acyltransferase, is essential for ghrelin to activate GHSR. ${ }^{16}$ Acylated ghrelin (AG, also called ghrelin or acyl-ghrelin) stimulates appetite and increases food intake by activating the vagal afferent neurons in the stomach via GHSR, whereas unacylated ghrelin (UAG, also called des-acyl ghrelin) does not. ${ }^{17}$ An

${ }^{1}$ Department of Internal Medicine, School of Medicine, Keio University, Tokyo, Japan. ${ }^{2}$ Division of Mucosal Immunology, Department of Microbiology and Immunology, The Institute of Medical Science, The University of Tokyo, Tokyo, Japan. ${ }^{3}$ International Research and Development Center for Mucosal Vaccines, The Institute of Medical Science, The University of Tokyo, Tokyo, Japan. ${ }^{4}$ Department of Polymer Chemistry, Graduate School of Engineering, Kyoto University, Kyoto, Japan. ${ }^{5} \mathrm{Japan}$ Science and Technology Agency (JST), The Exploratory Research for Advanced Technology (ERATO), Katura Int' Tech Center, Kyoto, Japan and 'Biochemicals Division, Yamasa Corporation, Chiba, Japan. Correspondence: T Azegami (t.azegami-1114@z2.keio.jp) or H Kiyono (kiyono@ims.u-tokyo.ac.jp) 
in vivo study has revealed that GHSR-null mice have a normal phenotype under standard diet conditions but are resistant to diet-induced obesity (DIO). ${ }^{18}$ Therefore, given its physiological function in weight gain and the fact that disrupting AG-GHSR signaling causes no severe adverse events and prevents weight gain, ghrelin is a potentially effective and safe target for antiobesity therapies.

Vaccination is a promising approach for the treatment of obesity because it has a prolonged therapeutic effect, needs a low frequency of administration, and is cost effective. Vaccination against ghrelin has been attempted in rat, pig, and mouse by using injectable peptide-based vaccines that required acylation of ghrelin antigen at serine 3 residue. ${ }^{19-22}$ Systemic immunization with these vaccines attenuated body weight gain in rat and pig $^{19,20}$ but did not in a DIO mouse model. ${ }^{21,22}$ Octanoyl modification of serine 3 residue of ghrelin is unstable and rapidly hydrolyzed in the body. ${ }^{23}$ Therefore, an antighrelin vaccine that can be administered non-invasively and that do not contain acylation at serine 3 residue may be useful.

As a non-invasive means of vaccine delivery, intranasal immunization offers distinct advantages over other injectable delivery methods such as decreased cost, less psychological and physiological stress and less risk of localized skin adverse events. ${ }^{24}$ Because intranasal administration of a protein-based antigen alone is not thought to be sufficient for the induction of antigen-specific immune responses, ${ }^{25}$ we previously developed an effective vaccine delivery system that uses a nanometer-sized hydrogel (nanogel) consisting of a cationic cholesteryl-groupbearing pullulan (cCHP) that effectively delivers antigens to antigen-presenting nasal dendritic cells. ${ }^{25}$ Nasal immunization with cCHP nanogel containing vaccine antigens (e.g., botulinum toxoid or pneumococcal surface protein A (PspA)) is able to deliver a vaccine antigen to the anionic nasal epithelium and induce both mucosal and systemic antigen-specific immune responses without accumulation of the vaccine antigens in the olfactory bulb or brain. ${ }^{25-27}$

Because ghrelin is a self-antigen to a vaccinated person, linkage of a carrier protein to self-antigen is essential for the sufficient induction of antigen-specific antibody production. However, there is currently no established carrier protein for nasal immunization because no nasal vaccine containing a recombinant peptide or protein is available for clinical use in humans. In animal studies, PspA, which is a cell surface protein expressed by all of the clinically important strains of Streptococcus pneumoniae, ${ }^{28}$ is a promising candidate antigen for use in recombinant protein-based nasal vaccines. A nanogel-based PspA vaccine has been shown to induce both systemic and mucosal antigen-specific protective immune responses while eliciting no serious adverse events, such as invasion of the vaccine antigen into the central nervous system in mouse and macaque. ${ }^{26,27}$ In the present study, PspA was thus selected as a carrier protein for the development of chimeric ghrelin vaccine.

Here we developed a novel nasal vaccine against obesity containing ghrelin as the vaccine antigen and cyclic di-GMP as the adjuvant and demonstrated that intranasal immunization with this vaccine successfully induced beneficial immune responses that attenuated body weight gain.

\section{RESULTS \\ Development of and immune responses induced by ghrelin-PspA}

We designed a novel vaccine antigen, ghrelin-PspA, which consists of three repeats of mouse UAG attached to the PspA sequence (see Supplementary Figure S1A online). GhrelinPspA plasmid was transfected into competent Escherichia coli cells and the purified protein was identified as ghrelin-PspA by means of western blotting (see Supplementary Figure S1B).

To confirm the immunogenicity of ghrelin-PspA, we intranasally immunized 4-week-old C57BL/6J mice with cCHP nanogel containing $5 \mu \mathrm{g}$ of ghrelin-PspA and $10 \mu \mathrm{g}$ of cyclic diGMP. Five times nasal vaccination induced serum immunoglobulin $\mathrm{G}$ (IgG) antibodies against $\mathrm{AG}$ and UAG (AG: reciprocal $\log _{2}$ titer $13.33 \pm 0.55$, UAG: $15.50 \pm 0.55, n=12$ per group). The main Ig isotype among the anti-AG serum antibodies was IgG; the production of other isotypes (i.e., IgM, $\operatorname{IgA}$, and $\mathrm{IgE}$ ) was scant (Figure 1a). After the start of immunization, the anti-AG serum IgG antibody titer rapidly
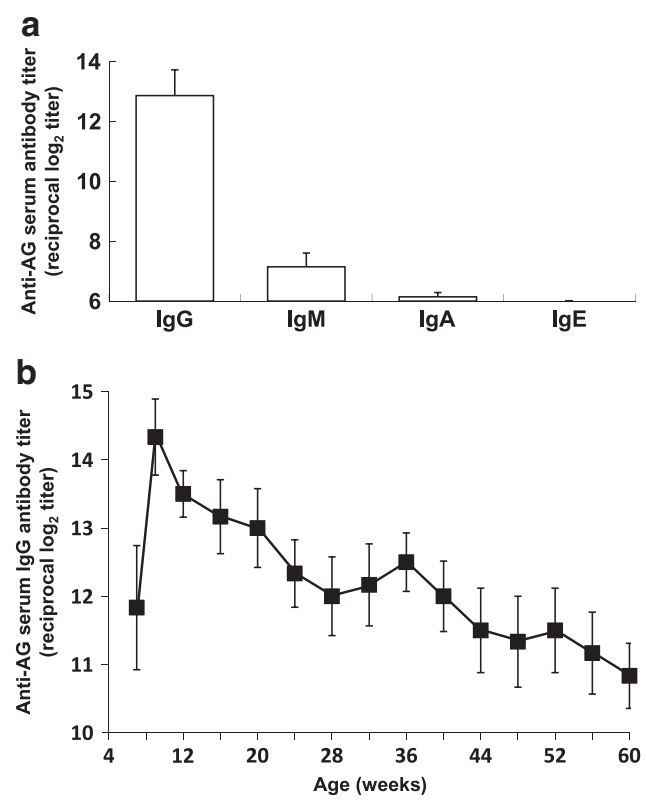

Figure 1 Intranasal immunization with ghrelin-PspA (pneumococcal surface protein A) vaccine induced antibodies against ghrelin and PspA. Sera and nasal washes were obtained from immunized mice after five times intranasal immunization with ghrelin-PspA vaccine, and antibody titers were determined by using enzyme-linked immunosorbent assays. (a) Serum immunoglobulin G (IgG) antibodies against acylated ghrelin (AG) were induced by immunization with ghrelin-PspA at 1 week after the final immunization. Other immunoglobulin isotypes (i.e., $\operatorname{lgM}$, $\lg A$, and $\lg \mathrm{E}$ ) against AG were scarce $(n=7)$. (b) Time course of the change in anti-AG serum IgG antibody titers in mice immunized with ghrelin-PspA vaccine at $4,5,6,7$, and 8 weeks of age and measured until 60 weeks of age (i.e., 52 weeks after the final vaccination). The antibody titer rapidly increased, reaching its peak at 1 week after the final immunization and then gradually decreased. However, a response was retained until 52 weeks after vaccination $(n=6)$. Data are presented as means \pm s.e.m. 
increased in mice, reaching its peak at 1 week after the final immunization ( 9 weeks of age). It then gradually decreased over several months, although a response was retained for $>1$ year (Figure 1b).

\section{Body weight gain, food intake, and energy expenditure after vaccination}

To evaluate the effect of ghrelin-PspA on body weight gain, we used two types of obesity model: the DIO mouse and the leptindeficient $(o b / o b)$ mouse. In the DIO mouse model, C57BL/6J mice were intranasally immunized five times with ghrelin-PspA and immediately after the final immunization a high-fat diet was initiated. Vaccination with ghrelin-PspA significantly attenuated weight gain in the DIO mice (Figure 2a). This weight
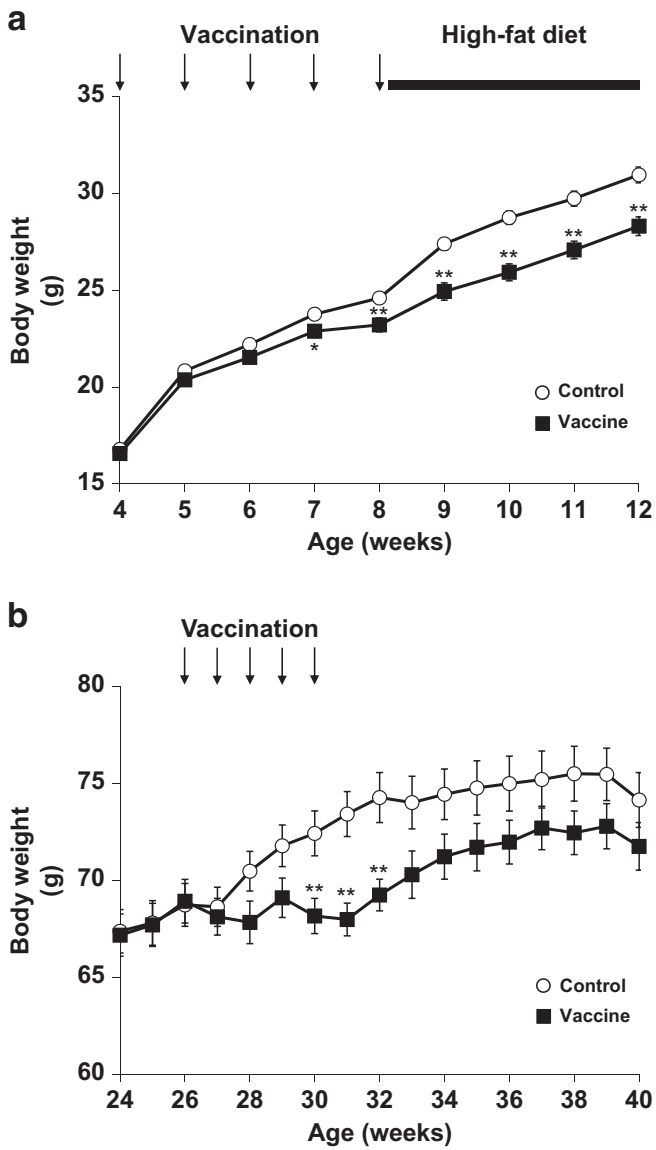

Figure 2 Intranasal immunization with ghrelin-PspA (pneumococcal surface protein A) vaccine attenuated body weight gain. Control and immunized mice were weighed every week. (a) Mice were intranasally immunized with or without ghrelin-PspA weekly at 4-8 weeks of age and then fed a high-fat diet ( $45 \%$ kcal from fat) after the final immunization. Body weight gain with growth was accelerated after initiation of the high-fat diet in control mice (control) and in immunized mice (vaccine). However, immunization with ghrelin-PspA vaccine significantly attenuated the body weight gain induced by the high-fat diet $(n=18)$. (b) Leptin-deficient genetically obese $(o b / o b)$ mice were fed a standard diet, and after the development of obesity, they were intranasally vaccinated weekly with ghrelin-PspA vaccine from 26 to 30 weeks of age. The body weight of control $o b / o b$ mice continued to increase, whereas that of immunized $o b /$ ob mice was significantly attenuated $(n=10)$. Data are presented as means \pm s.e.m., with differences between mean values evaluated for significance by using Student's $t$-test. ${ }^{\star} P<0.05$; ${ }^{*} P<0.01$. gain-attenuating effect persisted for 8 weeks after vaccination (control $34.8 \pm 0.7 \mathrm{~g}$ vs. vaccine $32.6 \pm 0.7 \mathrm{~g}$ at 8 weeks after the final immunization, $n=12$ per group, $P<0.05)$. Furthermore, intranasal administration of cyclic di-GMP adjuvant alone incorporated into the CCHP nanogel did not attenuate the dietinduced body weight gain (control $28.6 \pm 0.5 \mathrm{~g}$ vs. vehicle $28.0 \pm 0.6 \mathrm{~g}$ at 4 weeks, $n=12$ per group, $P=0.43$ ). In $o b / o b$ mice, body weight gain was also attenuated by immunization with ghrelin-PspA (Figure 2b). The changes in the body weight of $o b / o b$ mice between prevaccination and at 1 week after the final vaccination were significantly smaller than those of control ob/ob mice (control $4.7 \pm 0.3 \mathrm{~g}$ vs. ghrelin-PspA $-0.9 \pm 0.6 \mathrm{~g}, n=10$ per group, $P<0.01$; Figure $2 \mathbf{b}$ ).

To elucidate whether the attenuation in body weight gain in immunized DIO mice was due to a decrease in food intake or due to an increase in energy expenditure, we measured food intake and monitored oxygen consumption $\left(\mathrm{VO}_{2}\right)$ as an index of energy expenditure. Daily food intake did not differ between control and immunized DIO mice (Figure 3a). However, the
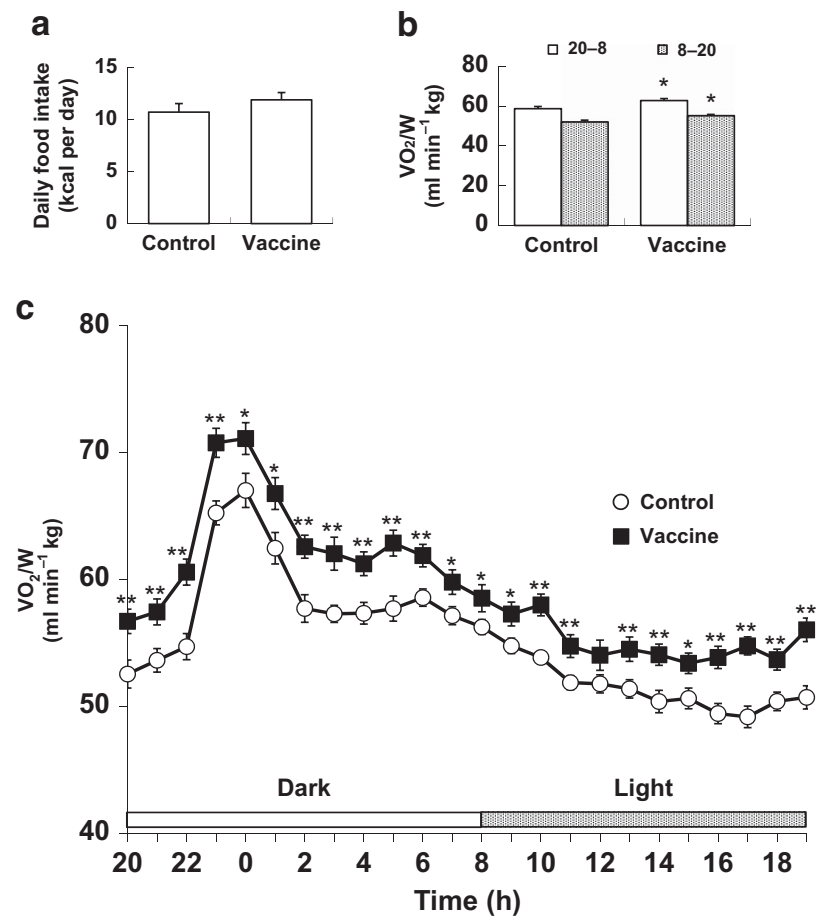

Figure 3 Intranasal immunization with ghrelin-PspA (pneumococcal surface protein A) vaccine did not alter food intake or body temperature but did increase energy expenditure. Food intake at 2-3 weeks and energy expenditure at 4 weeks after the final immunization were measured in control diet-induced obesity mice (control) and diet-induced obesity mice intranasally immunized weekly with ghrelin-PspA vaccine from 4 to 8 weeks of age (vaccine). (a) Individual daily food intake was measured on 2 consecutive days and the mean intake over the 2 days was defined as the daily food intake. There was no significant difference in daily food intake between the control and immunized mice $(n=12)$. (b, c) Oxygen consumption $\left(\mathrm{VO}_{2}\right)$ was measured by means of indirect calorimetry as an index of energy expenditure. Intranasal immunization with ghrelin-PspA vaccine significantly increased $\mathrm{VO}_{2}$ in both the active (dark) phase (20000800 hours) and the resting (light) phase (0800-2000 hours) $(n=9)$. Data are presented as means \pm s.e.m., with differences between mean values evaluated for significance by using Student's $t$-test. ${ }^{*} P<0.05$; ${ }^{\star \star} P<0.01$. 
respiratory gas analyses revealed that immunized DIO mice consumed more oxygen than did control DIO mice in both the active (dark) and resting (light) phase (Figure $3 \mathbf{b}, \mathbf{c}$ ). Rectal temperature, which is representative of systemic thermogenesis, was similar between the control and immunized DIO mice (control $37.0 \pm 0.4^{\circ} \mathrm{C}$ vs. vaccine $37.9 \pm 0.2^{\circ} \mathrm{C}, n=9$ per group, $P=0.686$ ). These data indicate that vaccination with ghrelin-PspA attenuated body weight gain by increasing energy expenditure.

\section{Visceral adiposity after immunization}

We also evaluated adiposity in DIO mice by using computed tomographic (CT) imaging and direct harvest at autopsy. Quantification of adiposity in the CT images revealed that the total amount of fat stored by vaccinated DIO mice was significantly lower than that stored by control DIO mice (Figure 4a). There were also significant reductions in visceral and subcutaneous fat (Figure 4a). The amount of visceral fat measured by means of direct harvest at autopsy was comparable with the results of the CT analysis (control $2.44 \pm 0.10 \mathrm{~g}$ vs. vaccine $1.53 \pm 0.08 \mathrm{~g}, n=18$ per group, $P<0.01$ ). Furthermore, the size of individual visceral adipocytes was also reduced in immunized mice compared with that in control mice (Figure 4b).

\section{Molecular and metabolic alterations in ghrelin-PspA-immunized mice}

Together, the data presented above suggest that the attenuation in body weight gain induced by immunization with ghrelinPspA was a result of increased energy expenditure reducing adiposity. Next, to evaluate the molecular regulation of energy expenditure in immunized DIO mice, the expression of mitochondrial uncoupling proteins (UCPs) and their upstream signals, peroxisome proliferator-activated receptors (PPARs), were quantified by means of real-time PCR (Figure 5a-i). In the brown adipose tissue (BAT), the expression of UCP1, which is responsible for nonshivering thermogenesis, was significantly increased in immunized mice compared with that in control mice (Figure 5a). The expression of PPAR $\gamma$, which is one of the upstream regulators of UCP1, was also significantly increased in the BAT of immunized mice compared with that in control mice (Figure 5d). In the white adipose tissue (WAT), the expression of UCP2 (Figure 5e) and PPAR $\gamma$ (Figure 5f) was significantly increased in immunized mice compared with that in control mice. In the muscle, the expression of UCP3, PPAR $\alpha$, and PPAR $\delta$ did not differ between control and immunized mice (Figure 5g-i).

In addition to the body weight-attenuating effect of immunization with ghrelin-PspA, we also evaluated the effect of immunization with the vaccine on glucose and lipid metabolism. Fasting blood glucose levels were comparable between control and immunized DIO mice (Figure 6a). However, the blood glucose levels of immunized DIO mice at $120 \mathrm{~min}$ after oral administration of glucose were significantly lower than those of control DIO mice (Figure 6a), suggesting that immunization with ghrelin-PspA improved glucose tolerance. Blood glucose levels after intraperitoneal administration of a
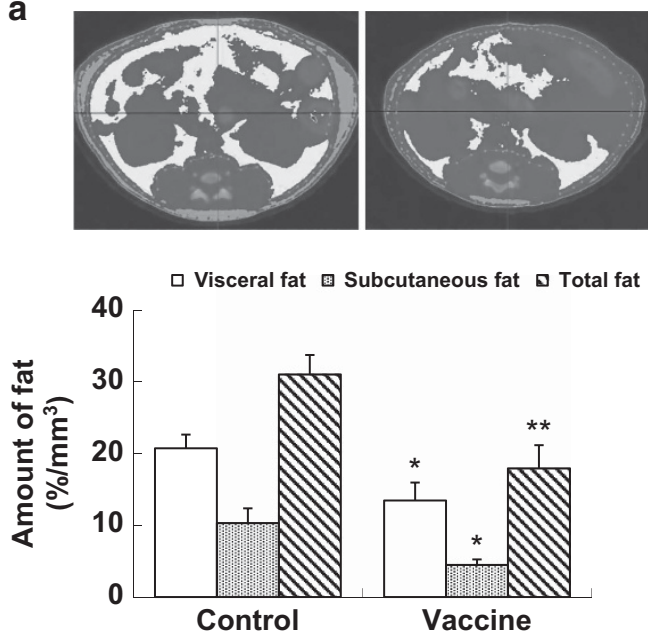

b
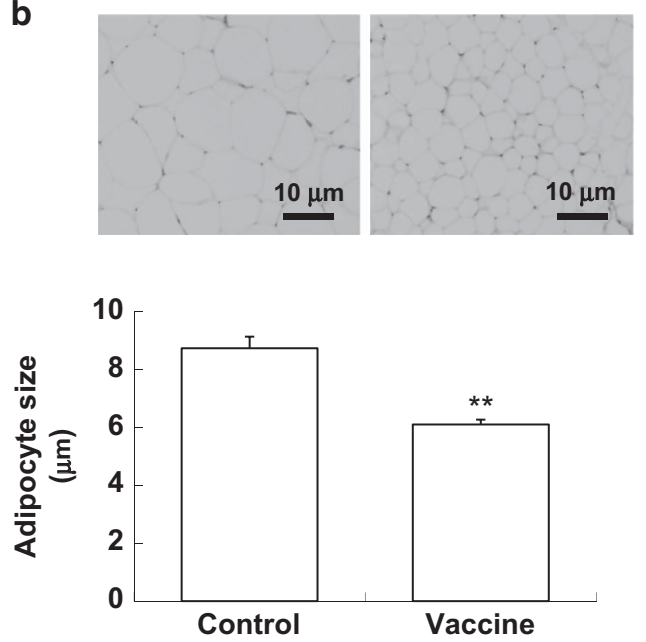

Figure 4 Intranasal immunization with ghrelin-PspA (pneumococcal surface protein $A$ ) vaccine attenuated fat accumulation and reduced adipocyte size. Control mice (control) and ghrelin-PspA vaccinated mice (vaccine) were fed a high-fat diet for 5 weeks. The adiposity was then analyzed in vivo by means of computer tomographic (CT) imaging. Perirenal adipose tissue was also collected and stained with hematoxylin and eosin for the measurement of adipocyte size. (a) CT scan was performed at the level of the fourth lumbar vertebra, and the resultant CT images were analyzed for the qualification of total, subcutaneous, and visceral fat. The amounts of total, subcutaneous, and visceral fat were significantly less in immunized diet-induced obesity (DIO) mice than in control DIO mice $(n=9)$. (b) Tissue samples of visceral fat were stained with hematoxylin and eosin, and adipocyte size was measured in five randomly selected fields as seen through a 100 -fold objective. The mean adipocyte size of immunized DIO mice was significantly less than that of control DIO mice $(n=9)$. Data are presented as means \pm s.e.m., with differences between mean values evaluated for significance by using Student's $t$-test. ${ }^{*} P<0.05$; ${ }^{\star \star} P<0.01$. A full color version of this figure is available at the Mucosal Immunology journal online.

insulin were also significantly lower in immunized DIO mice (Figure 6b), indicating that insulin resistance was attenuated by immunization with ghrelin-PspA. Fasting serum concentrations of free fatty acids, low-density lipoprotein, and triglycerides tended to be lower in immunized DIO mice compared with that in control DIO mice, but these differences were not significant (Figure 6c-e). 
a
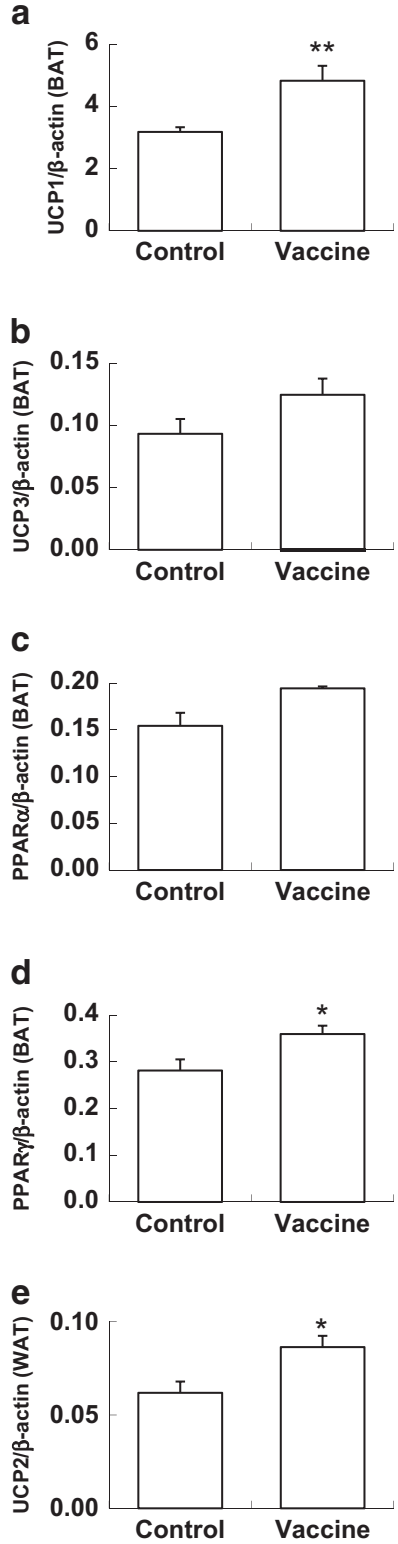

Figure 5 Intranasal immunization with ghrelin-PspA (pneumococca surface protein $A$ ) vaccine increased the expression of mitochondrial uncoupling protein (UCP) 1 and peroxisome proliferator-activated receptor gamma (PPAR $\gamma$ ) in brown adipose tissue (BAT) and of UCP2 and PPAR $\gamma$ in white adipose tissue (WAT). RNA was isolated from scapular fat (BAT), perirenal fat (WAT), and the quadriceps (skeletal muscle) of control diet-induced obesity (DIO) mice (control) and immunized DIO mice (vaccine) after 5 weeks' consumption of a high-fat diet. Isolated RNA was reverse transcribed and the resultant cDNA was quantitatively amplified by means of PCR. Relative gene expression data obtained by using realtime quantitative PCR was normalized to that of the gene encoding $\beta$-actin. (a-d) In BAT, intranasal vaccination with ghrelin-PspA vaccine significantly increased the expression of UCP1 and PPAR $\gamma$ but not that of UCP3 or PPAR $\alpha$. (e, f) In WAT, intranasal vaccination with ghrelin-PspA vaccine significantly increased the expression of UCP2 and PPAR $\gamma$. (g-i) In the skeletal muscle, intranasal vaccination with ghrelin-PspA vaccine did not alter the expression of UCP3, PPAR $\alpha$, and PPAR $\delta$ between control and immunized DIO mice. Data are presented as means \pm s.e.m., with differences between mean values evaluated for significance by using a Student's $t$-test. ${ }^{*} P<0.05 ;{ }^{\star *} P<0.01 ;(n=8)$.
Alterations in plasma levels of leptin, adiponectin, and growth hormone after immunization

In addition to ghrelin, leptin and adiponectin are also important hormones that mediate energy homeostasis. ${ }^{29}$ The plasma leptin and adiponectin levels of immunized DIO mice were comparable to those of control DIO mice (leptin: control $2.71 \pm 0.79 \mathrm{ng} \mathrm{ml}^{-1}$ vs. vaccine $1.86 \pm 0.53 \mathrm{ng} \mathrm{ml}^{-1}$, $P=0.283$; adiponectin: control $1779 \pm 221 \mathrm{ng} \mathrm{ml}^{-1}$ vs. vaccine $2944 \pm 902 \mathrm{ng} \mathrm{ml}^{-1}, P=0.227 ; n=9$ per group). Growth hormone is likely to be directly altered by modification of ghrelin-GHSR signaling because ghrelin is the endogenous ligand of $\mathrm{GHSR}^{13}$; however, the plasma level of growth hormone also did not significantly differ between control and immunized DIO mice (control $5.40 \pm 1.47 \mathrm{ng} \mathrm{ml}^{-1}$ vs. vaccine $9.51 \pm$ $4.16 \mathrm{ng} \mathrm{ml}^{-1}, n=9$ per group, $\left.P=0.499\right)$.

\section{Effect of vaccination-induced antibodies on endogenous ghrelin}

To confirm whether the IgG antibodies elicited by immunization with ghrelin-PspA directly inhibited the binding of AG to GHSR, we performed a receptor-binding assay. To obtain GHSR-expressing Chinese hamster ovary (CHO) cells, the DNA sequence encoding GHSR was synthesized and subcloned into the pcDNA3.1 $(+)$ vector; the resultant vector was then transfected into $\mathrm{CHO}$ cells (see Supplementary Figure S1C). We used a fluorescent indicator to measure the intracellular concentration of $\mathrm{Ca}^{2+}\left[\mathrm{iCa}^{2+}\right]$ before and after administration of AG with or without serum IgG obtained from either a control or immunized mouse. Administration of AG without serum IgG increased $\left[\mathrm{iCa}^{2+}\right.$ ] in GHSR-expressing $\mathrm{CHO}$ cells (Figure $7 \mathbf{a}, \mathbf{b})$. The addition of serum IgG obtained from control mice did not inhibit the increase in $\left[\mathrm{iCa}^{2+}\right]$, whereas the addition of serum IgG obtained from immunized mice did attenuate the increase in $\left[\mathrm{iCa}^{2+}\right]$ (Figure 7a,b), suggesting that AG-specific IgG antibodies directly inhibit AG-GHSR signaling.

\section{DISCUSSION}

Here we demonstrated a novel therapeutic means of intranasal immunization against obesity. Intranasal immunization with a newly developed vaccine, which contained the vaccine antigen ghrelin-PspA, attenuated body weight gain in DIO mice and leptin-deficient genetically obese mice. Most lifestylerelated diseases such as obesity are asymptomatic; therefore, non-invasive vaccination (e.g., mucosal administration) is preferable over invasive vaccination (e.g., subcutaneous or intramuscular injection) to increase medical adherence. The intranasal vaccine developed here provides advantages over injectable vaccines in terms of less pain, psychological stress, and local skin adverse events. ${ }^{24}$

Despite the lack of acylation at serine 3 residue of the ghrelin sequence in ghrelin-PspA antigen, intranasal immunization with ghrelin-PspA induced not only UAG-specific antibodies but also AG-specific serum IgG antibodies. In addition, immunization with ghrelin-PspA elicited polyclonal IgG antibodies against UAG (see Supplementary Figure S2A,B). The 

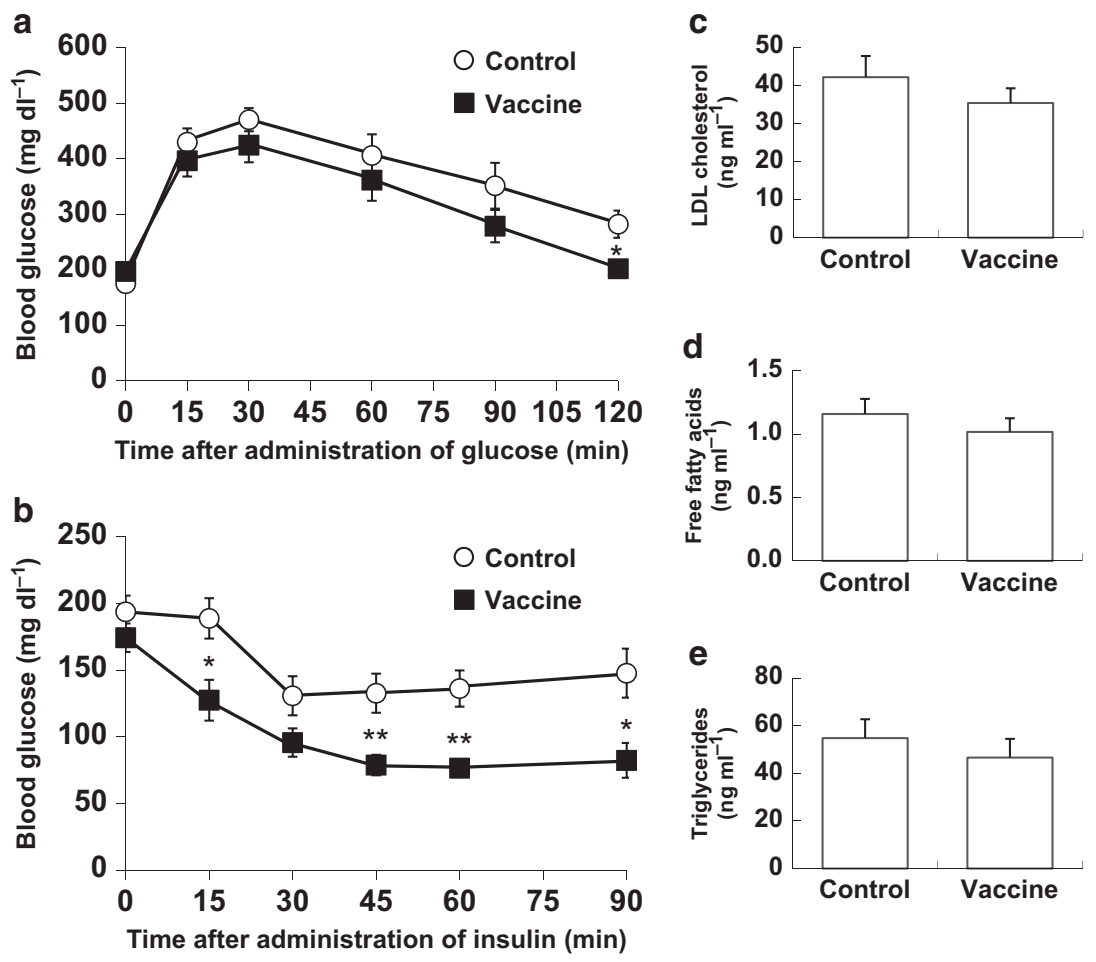

Figure 6 Intranasal immunization with ghrelin-PspA (pneumococcal surface protein A) vaccine attenuated glucose intolerance and improved insulin sensitivity but did not significantly change lipid metabolism. In control diet-induced (DIO) mice (control) and ghrelin-PspA immunized DIO mice (vaccine), oral glucose tolerance test (OGTT) and intraperitoneal insulin tolerance test (IPITT) were performed at 4 weeks after the final vaccination and the fasting serum concentrations of low-density lipoprotein (LDL) cholesterol, free fatty acids, and triglycerides were measured at 5 weeks after the final immunization. (a) In the OGTT, DIO mice fasted for $6 \mathrm{~h}$ were orally administered $2 \mathrm{~g}$ of glucose per kg body weight and their blood glucose levels were measured. At 120 min after the administration of glucose, the blood glucose levels of the vaccinated DIO mice were significantly lower than those of the control DIO mice $(n=9)$. (b) In IPITT, mice fasted for $6 \mathrm{~h}$ were intraperitoneally administered $0.75 \mathrm{IU}$ of insulin per kg body weight and their blood glucose levels were measured. After injection of insulin, the blood glucose levels of the immunized DIO mice were significantly lower than those of the control DIO mice $(n=9)$. (c-e) The fasting serum concentrations of LDL, free fatty acids, and triglycerides were slightly lower in immunized DIO mice than in control DIO mice, but these differences were not statistically significant $(n=9)$. Data are presented as means $\pm \mathrm{s}$.e.m., with differences between mean values evaluated for significance by using Student's $t$-test. ${ }^{\star} P<0.05$; ${ }^{\star \star} P<0.01$.

main targets of the induced serum IgG antibodies were the $\mathrm{N}$-terminal (amino acids 1-9) and the C-terminal (amino acids 20-28) portions of the ghrelin sequence (see Supplementary Figure S2A,B), as shown by enzyme-linked immunosorbent assay (ELISA), which is thought to be why immunization with ghrelin-PspA elicited antibodies against both AG and UAG.

In terms of the immunization schedule, we intranasally administered ghrelin-PspA vaccine once a week for 5 weeks. One or two doses of vaccine did not induce AG-specific antibody responses (see Supplementary Figure S3). Although serum IgG antibody against AG was elicited when intranasal immunization was given three or four times, five doses of immunization induced a higher anti-AG antibody titer (see Supplementary Figure S3). In addition, three doses of nasal vaccination with ghrelin-PspA vaccine did not ameliorate the body weight gain in DIO mice (data not shown). Therefore, we concluded that a protocol of five doses of intranasal immunization was the most suitable for attenuating obesity.

Although a high titer for antibodies against AG was maintained at 1 year after five doses of immunization, the effect on the attenuation of body weight gain was lost at 9 weeks after the final vaccination in DIO mice. There are two possible explanations for this discrepancy. The first is simply that the antibody titer at 9 weeks after the final immunization (reciprocal $\log _{2}$ titer approximately 13) was functionally insufficient compared with the titers in the previous weeks (reciprocal $\log _{2}$ titer at 1 week, 14.33; at 8 weeks, 13.17 ; and at 12 weeks after vaccination, 13.00) (Figure 1b). Indeed, in a preliminary study where intranasal immunization was given only three times, not five times, ghrelin-PspA induced an increased antibody titer (max. reciprocal $\log _{2}$ titer 11.83 ) but did not attenuate body weight gain in DIO mice (data not shown). The second possibility is that the weight gain in DIO mice might become accelerated or uncontrolled after 9 weeks of being fed a high-fat diet, meaning that the AG-specific antibody alone is insufficient to attenuate the body weight gain. Indeed, it has been shown that long-term consumption of a high-fat diet in mice has the potential to cause refractory obesity that cannot be fully reversed by reducing the amount of fat in the diet. ${ }^{30}$

Intranasal immunization with ghrelin-PspA had attenuated the body weight gain in DIO mice by $9.5 \%$ compared with that in control DIO mice at 4 weeks of being fed a high-fat diet ( $45 \% \mathrm{kcal}$ from fat). This was consistent with a previous study showing that male GHSR-null mice weighed $10.9 \%$ less than wild-type C57BL/6 mice after 6 weeks of being fed a high-fat diet $\left(42 \% \mathrm{kcal}\right.$ from fat). ${ }^{18}$ In addition to its preventive effect 
a

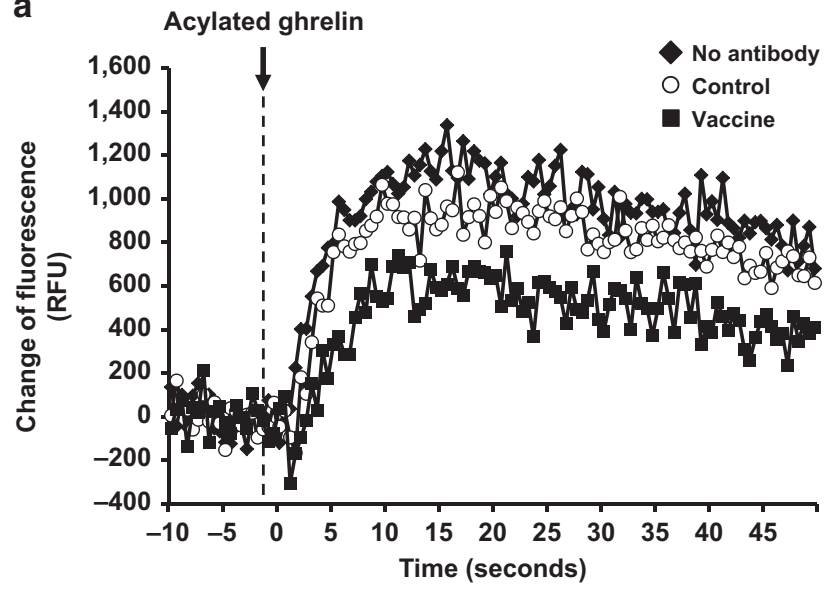

b

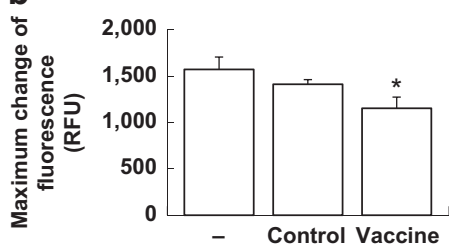

Figure 7 Serum immunoglobulin G ( $\operatorname{lgG}$ ) antibodies induced by intranasal immunization with ghrelin-PspA (pneumococcal surface protein $A$ ) vaccine directly inhibited the binding of acylated ghrelin (AG to its receptor, growth hormone secretagogue receptor (GHSR). Serum polyclonal IgG antibodies were obtained from either control mice (control) or mice intranasally immunized five times with ghrelin-PspA vaccine (vaccine). The intracellular concentration of $\mathrm{Ca}^{2+}$ in GHSR-expressing Chinese hamster ovary $(\mathrm{CHO})$ cells, which is a downstream signal of ghrelin receptor activation, was measured before and after the administration of $A G$ with or without serum IgG by using a fluorescent indicator that binds to $\mathrm{Ca}^{2+}$. (a) Administration of $A G$ alone to GHSRexpressing $\mathrm{CHO}$ cells caused a rapid increase in intracellular fluorescence (i.e., an increase in intracellular $\mathrm{Ca}^{2+}$ concentration). When $\mathrm{AG}$ and IgG antibodies obtained from immunized mice were administered to GHSRexpressing $\mathrm{CHO}$ cells, the increase in the intracellular concentration of $\mathrm{Ca}^{2+}$ was attenuated. In contrast, the addition of IgG antibodies obtained from control mice did not alter the change in the intracellular concentration of $\mathrm{Ca}^{2+}$. (b) The maximum change in the intracellular concentration of $\mathrm{Ca}^{2+}$ between before and after administration of $A G$ was significantly suppressed when IgG antibodies of immunized mice, but not of control mice, were added. Data are presented as means \pm s.e.m., with differences between mean values evaluated for significance by using Student's $t$-test. ${ }^{*} P<0.05 ;(n=8)$.

against diet-induced body weight gain, intranasal immunization with ghrelin-PspA also decreased body weight in a leptindeficient genetically obese mouse (i.e., $o b / o b$ mouse).

Ghrelin-PspA vaccine was found to not alter food intake in immunized DIO mice, even though ghrelin is known to stimulate appetite. ${ }^{17}$ However, it should be noted that the effect of the ablation of ghrelin-GHSR signaling on food intake is controversial. A previous study has revealed that GHSR-null mice demonstrated lower food intake than did wild-type mice; ${ }^{18}$ however, other studies have shown that there is no difference in food intake between wild-type and GHSR-null mice. ${ }^{31,32}$ Some previously reported antighrelin peptide-based vaccines have been shown to reduce food intake in pigs and mice fed standard chow, ${ }^{20,22}$ but others have not shown a change in food consumption in rats and mice fed a high-fat diet. $^{19,21,22}$ In view of the lack of a decrease in the amount of food consumed in the present study, vaccination with ghrelinPspA may be more effective when used in combination with dietary restriction, although further examination is required.

In contrast to the results regarding food intake, the results obtained regarding the role of ghrelin inhibition in energy expenditure were in good agreement with those reported from previous studies, confirming that the absence of ghrelin action elicits increased energy expenditure. ${ }^{18,31,32}$ In the present study, immunization with ghrelin-PspA increased energy expenditure and UCP1 expression in BAT. UCP1 mediates proton leak in mitochondria, which is a process that facilitates the dissipation of metabolic fuels, resulting in the release of heat and control of energy expenditure. ${ }^{33}$ Therefore, increased UCP1 expression in BAT is likely to be responsible, at least in part, for the increased energy expenditure seen in the mice immunized with ghrelin-PspA. In fact, central administration or chronic intraperitoneal injection of ghrelin has been shown to decrease the expression of UCP1 in BAT, ${ }^{34,35}$ resulting in increased body weight and adiposity. In contrast, GHSR antagonists have been shown to increase the expression of $\mathrm{UCP} 1 .{ }^{36} \mathrm{UCP} 1$ is regulated by PPAR $\alpha$ and PPAR $\gamma$ and their upstream signals, such as sympathetic nervous system activation and fatty acids. ${ }^{37} \mathrm{~A}$ previous study demonstrated that central administration of ghrelin attenuated sympathetic nerve activity in BAT. ${ }^{38}$ In the present study, inhibition of ghrelin by immunization with ghrelin-PspA increased PPAR $\gamma$ expression in BAT. These findings likely explain the increased expression of UCP1 in immunized mice.

Previous studies have reported that ghrelin increases PPAR $\gamma$ production in $\mathrm{WAT}^{39}$ and that it does not alter PPAR $\gamma$ expression in WAT. $^{40}$ In the present study, intranasal administration of ghrelin-PspA significantly increased the expression of PPAR $\gamma$ in WAT in DIO mice. PPAR $\gamma$ is a key regulator for UCP2 in WAT. ${ }^{41}$ Although few studies have examined the role of ghrelin in the expression of UCP2 in WAT, chronic peripheral administration of ghrelin has been shown to result in increased production of UCP $2,{ }^{35}$ which is inconsistent with our results. Although the physiological role of UCP2 remains unclear, it does not appear to regulate energy expenditure and thermogenesis. ${ }^{33}$ Therefore, although the physiological reason for the increase in the expression of PPAR $\gamma$ and UCP2 in WAT in mice immunized with ghrelinPspA remains unclear, the contribution of these proteins to the attenuation of body weight gain is thought to be minimal.

In addition to molecular alterations in adipose tissue, both the total amount of adipose tissue and the size of individual adipocytes were significantly decreased by immunization with ghrelin-PspA, which is consistent with the finding of a previous study showing that GHSR-null mice fed a high-fat diet showed reduced fat accumulation. ${ }^{31}$ Smaller adipocytes are associated with high insulin sensitivity and improved glucose tolerance; ${ }^{42}$ therefore, the reduction of adipocyte size in the immunized mice may account for the improved results observed for the oral glucose tolerance test and intraperitoneal insulin tolerance test. As discussed above, PPAR $\gamma$ expression in adipocytes was 
increased by immunization with ghrelin-PspA. PPAR $\gamma$ in adipose tissue is known to have a role in preventing the adipocyte hypertrophy and insulin resistance induced by a high-fat diet. ${ }^{43}$ Taken together, the increased energy expenditure in the immunized mice appears to change the quality and quantity of adipose tissue, that is, it decreases the size of adipocytes, reduces fat mass, and increases PPAR $\gamma$ expression, which together improves glucose metabolism.

Immunization with ghrelin-PspA not only elicited serum IgG antibodies against $A G$ and UAG but also increased the plasma concentrations of AG and UAG (see Supplementary Figure S4A,B). In previous reports, immunization with antighrelin vaccines has been shown to increase plasma AG concentration. ${ }^{21,22}$ However, in the present study, the expression of ghrelin and GHSR mRNA in the stomach was not altered by immunization with ghrelin-PspA vaccine (see Supplementary Figure S4C,D), suggesting that ghrelinPspA blocks ghrelin-GHSR signaling but does not induce the production of ghrelin and the expression of ghrelin receptor. In addition, deficiency of GHSR has been shown to not alter plasma AG concentration in mice and administration of ghrelin $\mathrm{O}$-acyltransferase inhibitor has been shown to not increase plasma UAG concentration in mice, ${ }^{44,45}$ suggesting that the increased plasma concentrations of AG and UAG induced by immunization with ghrelin-PspA was not due to feedback from inhibition of AG-GHSR signaling. Therefore, a possible reason for the increase in circulating AG and UAG in the immunized mice is prolongation of the half-life of ghrelin owing to the binding of antibodies to the AG and UAG. In fact, immunization with ghrelin-PspA prolonged the half-life of AG in vivo (control $51.3 \mathrm{~min}$ vs. vaccine $89.6 \mathrm{~min}, n=3$ per group, see Supplementary Figure S5A). Moreover, gel filtration chromatographic analysis of plasma samples revealed that the fraction containing the most plasma IgG also contained the most AG (see Supplementary Figure S5B), indicating that the excess ghrelin detected in the plasma of immunized mice may actually be AG-specific IgG bound to AG, whose half-life is prolonged because of interference with the elimination of IgGAG complex from the body. As plasma concentrations of UAG were increased in immunized mice, there is the possibility that the attenuation of body weight gain induced by immunization with ghrelin-PspA was caused by increased circulating UAG. Although there are conflicting reports concerning the effects of UAG on food intake, ${ }^{46,47}$ transgenic mice overexpressing UAG have been shown to exhibit reduced body weight. ${ }^{48}$ As UAG is not supposed to bind to or activate $\mathrm{AG}$ receptor (i.e., GHSR ${ }^{13}$ and no specific receptor for UAG has been identified, it would be difficult here to discuss the involvement of UAG in the obesity-attenuating effect of immunization with ghrelin-PspA. Therefore, we evaluated the direct interaction between ghrelin and ghrelin-specific IgG antibodies induced by immunization with ghrelin-PspA. A receptor assay using GHSR-expressing $\mathrm{CHO}$ cells demonstrated that AG was directly inhibited from binding to GHSR by serum IgG antibodies induced by immunization with ghrelin-PspA and therefore that the downstream signals of AG-GHSR (i.e., $\left[\mathrm{iCa}^{2+}\right]$ ) were also blocked.
To examine the safety of immunization with ghrelin-PspA with regard to the induction of excess autoimmune responses, we histologically observed two organs that are known to express ghrelin: the stomach and the brain. Under light microscopy, no accumulation or infiltration of lymphocytes was observed in the gastric fundal mucosa or hypothalamic arcuate nucleus, which are the locations where ghrelin is mainly produced (see Supplementary Figure S6A,B). Moreover, the concentrations of circulating pro-inflammatory cytokines interleukin (IL)-1 $\beta$, IL-6, and tumor necrosis factor $\alpha$ were not increased in immunized mice (see Supplementary Figure S6C-E), suggesting a lack of non-specific or excess systemic immune responses.

As shown above, the novel intranasally administered ghrelinPspA vaccine developed here has the potential to prevent and attenuate obesity. The concept for nasal vaccination against human common disorders (not only communicable but also noncommunicable diseases) is a novel and creative challenge for the development of future human vaccines. Nasal vaccination is a promising approach for the treatment of chronic diseases because it has a prolonged therapeutic effect, needs a low frequency of administration, and avoids pain and local skin adverse events. Cumulatively, our findings show that intranasal immunization with ghrelin-PspA vaccine attenuates body weight gain without the need for invasive injections in mice, suggesting an innovative strategy for vaccination against human lifestyle-related disorder.

\section{METHODS}

Animals. Male C57BL/6J Jcl mice were obtained from CLEA Japan (Tokyo, Japan), and male C57BL/6J Ham Slc-ob/ob mice were obtained from Japan SLC (Hamamatsu, Japan). All mice were housed with ad libitum food and water under standard 12-h light/12-h dark conditions according to the guidelines provided by the Animal Experiment Committee of the Institute of Medical Science of The University of Tokyo. The mice were fed either standard pellet chow (CA-1; CLEA Japan) or a high-fat diet (D12451; Research Diets, New Brunswick, NJ) with metabolizable energy contents of $3.47 \mathrm{kcal} \mathrm{g}^{-1}$ (13.0\% kcal from fat) and $4.73 \mathrm{kcal} \mathrm{g}^{-1}$ (45\% kcal from fat), respectively. DIO mice were fed a standard diet until the final immunization at 8 weeks of age and a high-fat diet thereafter, and $o b / o b$ mice were fed standard chow throughout the whole experiment.

Synthesis of recombinant ghrelin-PspA protein. Ghrelin-PspA vaccine antigen comprised three repeats of mouse whole UAG at the C-terminus and the PspA sequence at the N-terminus. Ghrelin-PspA protein was identified in the western blottings by using antighrelin, anti-PspA (previously produced by immunization with PspA to mice) ${ }^{26}$ and anti-His-tag primary antibodies. Details are provided in Supplementary Materials and Methods.

Preparation of the nanogel-based ghrelin-PspA vaccine and immunization protocol. To enhance the antigen-specific immune responses, $10 \mu \mathrm{g}$ of cyclic di-GMP (produced by Yamasa Corporation, Choshi, Japan) was added to $5 \mu \mathrm{g}$ of ghrelin-PspA protein. cCHP nanogel, which was synthesized as described previously, ${ }^{25}$ was then mixed at a 3:1 molecular ratio with ghrelin-PspA and incubated for $1 \mathrm{~h}$ at $46^{\circ} \mathrm{C}$ to incorporate the vaccine antigen and adjuvant into cationiccharged nanoparticles.

Mice were intranasally immunized with ghrelin-PspA vaccine (total volume, $7.99 \mu$ l per mouse) on five occasions at 1-week intervals (at 4, 5, 6, 7, and 8 weeks of age in DIO mice and at 26, 27, 28, 29, and 30 weeks of age in $o b / o b$ mice) by using a micropipette to deliver the fluid dropwise into each nostril. 
Antibody titers. Serum antibody titers were determined by using ELISAs. Each well of a 96-micro-well plate (Immulon $1 \mathrm{~B}$; Thermo Fisher Scientific, Waltham, MA) was coated with $0.1 \mu \mathrm{g}$ of AG, UAG, or partial UAG peptides conjugated with bovine serum albumin. Diluted samples of serum were loaded into individual wells of plates. The end point titer was expressed as the reciprocal $\log _{2}$ of the last dilution that gave an $\mathrm{OD}_{450}$ that was 0.1 units greater than that of the negative control. Details are provided in Supplementary Materials and Methods.

Body weight, food intake, and body temperature of the mice. All mice were weighed once each week by using a precision balance (GX6000; A\&D, Tokyo, Japan) with $0.1 \mathrm{~g}$ precision. Individual daily food intake was measured on 2 consecutive days at 2-3 weeks after the final vaccination and the mean was defined as the daily food intake. The body temperature of the mice was measured rectally at 4-5 weeks after the last immunization by using a digital thermometer with a rectal probe (BDT-100, Bio Research Center, Nagoya, Japan).

Respiratory gas analyses. Energy expenditure was assessed by means of indirect calorimetry by calculating $\mathrm{VO}_{2}$ at 4 weeks after the final vaccination ( $n=9$ per group). Details are provided in Supplementary Materials and Methods.

Quantification and qualification of visceral adiposity. To obtain CT images, DIO mice ( 5 weeks after the final immunization; $n=9$ per group) were anesthetized in a sealed chamber containing $1 \%$ isoflurane and then fixed in the supine position under continuous isoflurane inhalation. CT scans were performed with an in vivo three-dimensional micro X-ray CT (R_mCT2; Rigaku Corporation, Tokyo, Japan) (voltage, $100 \mathrm{kV}$; current, $160 \mu \mathrm{A}$ ) at the level of the fourth lumbar vertebra. The visceral and subcutaneous fat seen in the CT images were quantified by using the CTAtlas Metabolic Analysis Ver. 2.03 software (Rigaku Corporation). In addition to the CT analysis, the total amount of mesenteric, epididymal, and perirenal fat, as a visceral fat, was also directly measured by harvesting at autopsy. Harvested perirenal fat was stained with hematoxylin and eosin as described below, and adipocyte size was measured with Adobe Photoshop CS4 (Adobe Systems Incorporated, San Jose, CA) by randomly selecting 5 fields with a 100fold objective for each mouse ( $n=9$ per group).

Histological studies. Tissue samples of WAT (perirenal fat), stomach, and brain were fixed in $4 \%$ paraformaldehyde and embedded in paraffin blocks. The hypothalamus in the brain tissue was serially sectioned in the coronal plane. The stomach tissue was sectioned vertically. All tissue sections were stained with hematoxylin and eosin. Brain and stomach sections were examined to evaluate whether or not inflammatory cells had infiltrated the tissue.

Quantitative real-time reverse transcription PCR. Total RNAs from BAT, WAT, and skeletal muscle were quantified by real-time reverse transcription PCR. Details are provided in Supplementary Materials and Methods. The oligonucleotides used for the PCR are listed in Supplementary Table S1.

Glucose and lipid homeostasis. Oral glucose tolerance test and intraperitoneal insulin tolerance test were performed at 4 weeks after the final vaccination ( $n=9$ per group). Details are provided in Supplementary Materials and Methods. For evaluation of lipid metabolism, the concentrations of low-density lipoprotein, free fatty acids, and triglycerides in fasting sera were measured by means of an enzymatic colorimetric assay (Wako Pure Chemical Industries, Osaka, Japan) at 5 weeks after the final vaccination ( $n=9$ per group).

Measurement of the plasma concentrations of ghrelin, leptin, adiponectin, and growth hormone. Concentrations of AG, UAG, leptin, adiponectin, and growth hormone in plasma obtained from control and immunized DIO mice fasted for $16 \mathrm{~h}$ at 5 weeks after the final vaccination were measured by double-antibody sandwich technique (SPI-Bio, Montigny Le Bretonneux, France) or ELISA methods (EMD Millipore Corporation, Billerica, MA) ( $n=9$ per group). Half-life of AG was determined from the radioactive decay rate of ${ }^{111}$ In. Details are provided in Supplementary Materials and Methods.

To confirm that AG bound to IgG antibody could be measured with a sandwich enzyme immunometric assay, a fasting plasma sample of the immunized mouse was separated by means of gel filtration using a Sephacryl S100 column (GE Healthcare Bio-Sciences K.K., Tokyo, Japan). Peak AG-containing fractions were evaluated by means of western blotting using an anti-mouse IgG antibody (1:5000, Jackson ImmunoResearch Laboratories, West Grove, PA).

Evaluation of the role of antibodies induced by immunization. GHSR-expressing CHO cells were obtained by using Lipofectamine 3000 Transfection Reagent (Thermo Fisher Scientific). Changes in $\left[\mathrm{iCa}^{2+}\right]$ in GHSR-expressing $\mathrm{CHO}$ cells after administration of AG with or without serum IgG antibodies were measured by using a Calcium Kit Fluo-4 (Dojindo Laboratories, Kumamoto, Japan). Details are provided in Supplementary Materials and Methods.

Non-specific systemic inflammatory responses. The concentrations of systemic IL-1 $\beta$, IL- 6 , and tumor necrosis factor $\alpha$ in sera obtained from C57/BL6J mice with or without intranasal vaccination with ghrelin-PspA at 2 weeks after the last immunization were determined by using the sandwich ELISA (Abcam, Cambridge, UK) according to the manufacturer's protocol ( $n=6$ per group).

Statistical analysis. Data are expressed as mean \pm s.e.m. Statistical comparisons were made by analysis of variance followed by an unpaired two-tailed Student's $t$-test between the two groups. $P$-values of $<0.01$ and $<0.05$ were considered to be statistically significant.

SUPPLEMENTARY MATERIAL is linked to the online version of the paper at http://www.nature.com/mi

\section{ACKNOWLEDGMENTS}

This study was supported by grants from the AMED-CREST, Japan Agency for Medical Research and Development, the Research on Development of New Drugs program of the Japan Agency for Medical Research and Development, and the Mochida Memorial Foundation for Medical and Pharmaceutical Research.

\section{AUTHOR CONTRIBUTIONS}

T.A. conducted the research, performed the experiments, and wrote the manuscript. Y.Y., H.I. and H.K. supervised the project and planned the research. S.S., M.M., K.I. and K.A. provided technical support.

\section{DISCLOSURE}

The authors declared no conflict of interest.

c) 2017 Society for Mucosal Immunology

\section{REFERENCES}

1. Global Burden of Metabolic Risk Factors for Chronic Diseases Collaboration et al. Metabolic mediators of the effects of body-mass index, overweight, and obesity on coronary heart disease and stroke: a pooled analysis of 97 prospective cohorts with 1.8 million participants. Lancet 383, 970-983 (2014).

2. Ng, M. et al. Global, regional, and national prevalence of overweight and obesity in children and adults during 1980-2013: a systematic analysis for the Global Burden of Disease Study 2013. Lancet 384, 766-781 (2014).

3. Wang, Y.C., McPherson, K., Marsh, T., Gortmaker, S.L. \& Brown, M. Health and economic burden of the projected obesity trends in the USA and the UK. Lancet 378, 815-825 (2011).

4. Leblanc, E.S., O'Connor, E., Whitlock, E.P., Patnode, C.D. \& Kapka, T. Effectiveness of primary care-relevant treatments for obesity in adults: a systematic evidence review for the U.S. Preventive Services Task Force. Ann. Intern. Med. 155, 434-447 (2011). 
5. Smith, S.R. et al. Multicenter, placebo-controlled trial of lorcaserin for weight management. N. Engl. J. Med. 363, 245-256 (2010).

6. Gadde, K.M. et al. Effects of low-dose, controlled-release, phentermine plus topiramate combination on weight and associated comorbidities in overweight and obese adults (CONQUER): a randomised, placebocontrolled, phase 3 trial. Lancet 377, 1341-1352 (2011).

7. Greenway, F.L. et al. Effect of naltrexone plus bupropion on weight loss in overweight and obese adults (COR-I): a multicentre, randomised, doubleblind, placebo-controlled, phase 3 trial. Lancet 376, 595-605 (2010).

8. Pi-Sunyer, X. et al. A randomized, controlled trial of $3.0 \mathrm{mg}$ of liraglutide in weight management. N. Engl. J. Med. 373, 11-22 (2015).

9. Filippatos, T.D., Derdemezis, C.S., Gazi, I.F., Nakou, E.S., Mikhailidis, D.P. \& Elisaf, M.S. Orlistat-associated adverse effects and drug interactions: a critical review. Drug Saf. 31, 53-65 (2008).

10. Melia, A.T., Koss-Twardy, S.G. \& Zhi, J. The effect of orlistat, an inhibitor of dietary fat absorption, on the absorption of vitamins $A$ and $E$ in healthy volunteers. J. Clin. Pharmacol. 36, 647-653 (1996).

11. Halpern, B. \& Halpern, A. Safety assessment of FDA-approved (orlistat and lorcaserin) anti-obesity medications. Expert Opin. Drug Saf. 14, 305-315 (2015).

12. Tschop, M., Smiley, D.L. \& Heiman, M.L. Ghrelin induces adiposity in rodents. Nature 407, 908-913 (2000).

13. Kojima, M., Hosoda, H., Date, Y., Nakazato, M., Matsuo, H. \& Kangawa, K. Ghrelin is a growth-hormone-releasing acylated peptide from stomach. Nature 402, 656-660 (1999).

14. Date, Y. et al. Ghrelin, a novel growth hormone-releasing acylated peptide, is synthesized in a distinct endocrine cell type in the gastrointestinal tracts of rats and humans. Endocrinology 141, 4255-4261 (2000).

15. Gnanapavan, S. et al. The tissue distribution of the mRNA of ghrelin and subtypes of its receptor, GHS-R, in humans. J. Clin. Endocrinol. Metab. 87 2988 (2002)

16. Yang, J., Brown, M.S., Liang, G., Grishin, N.V. \& Goldstein, J.L. Identification of the acyltransferase that octanoylates ghrelin, an appetite-stimulating peptide hormone. Cell 132, 387-396 (2008).

17. Date, Y. et al. The role of the gastric afferent vagal nerve in ghrelin-induced feeding and growth hormone secretion in rats. Gastroenterology 123 , 1120-1128 (2002).

18. Zigman, J.M. et al. Mice lacking ghrelin receptors resist the development of diet-induced obesity. J. Clin. Invest. 115, 3564-3572 (2005).

19. Zorrilla, E.P. et al. Vaccination against weight gain. Proc. Natl. Acad. Sci. USA 103, 13226-13231 (2006).

20. Vizcarra, J.A., Kirby, J.D., Kim, S.K. \& Galyean, M.L. Active immunization against ghrelin decreases weight gain and alters plasma concentrations of growth hormone in growing pigs. Domest. Anim. Endocrinol. 33, 176-189 (2007).

21. Kellokoski, E. et al. Ghrelin vaccination decreases plasma MCP-1 level in LDLR(-/-)-mice. Peptides 30, 2292-2300 (2009).

22. Andrade, $\mathrm{S}$. et al. Immunization against active ghrelin using virus-like particles for obesity treatment. Curr. Pharm. Des 19, 6551-6558 (2013).

23. Hosoda, H. \& Kangawa, K. Standard sample collections for blood ghrelin measurements. Methods Enzymol. 514, 113-126 (2012).

24. Lamichhane, A., Azegamia, T. \& Kiyono, H. The mucosal immune system for vaccine development. Vaccine 32, 6711-6723 (2014).

25. Nochi, T. et al. Nanogel antigenic protein-delivery system for adjuvant-free intranasal vaccines. Nat. Mater. 9, 572-578 (2010).

26. Kong, I.G. et al. Nanogel-based PspA intranasal vaccine prevents invasive disease and nasal colonization by Streptococcus pneumoniae. Infect. Immun. 81, 1625-1634 (2013).

27. Fukuyama, Y. et al. Nanogel-based pneumococcal surface protein $A$ nasal vaccine induces microRNA-associated Th17 cell responses with neutralizing antibodies against Streptococcus pneumoniae in macaques. Mucosal Immunol 8, 1144-1153 (2015).

28. Crain, M.J. et al. Pneumococcal surface protein A (PspA) is serologically highly variable and is expressed by all clinically important capsular serotypes of Streptococcus pneumoniae. Infect. Immun. 58, 3293-3299 (1990).

29. Badman, M.K. \& Flier, J.S. The gut and energy balance: visceral allies in the obesity wars. Science 307, 1909-1914 (2005).

30. Guo, J., Jou, W., Gavrilova, O. \& Hall, K.D. Persistent diet-induced obesity in male C57BL/6 mice resulting from temporary obesigenic diets. PLoS One 4, e5370 (2009).

31. Wortley, K.E. et al. Absence of ghrelin protects against early-onset obesity. J. Clin. Invest. 115, 3573-3578 (2005).

32. Pfluger, P.T. et al. Simultaneous deletion of ghrelin and its receptor increases motor activity and energy expenditure. Am. J. Physiol. Gastrointest. Liver Physiol. 294, G610-G618 (2008).

33. Krauss, S., Zhang, C.Y. \& Lowell, B.B. The mitochondrial uncouplingprotein homologues. Nat. Rev. Mol Cell. Biol. 6, 248-261 (2005).

34. Theander-Carrillo, C. et al. Ghrelin action in the brain controls adipocyte metabolism. J. Clin. Invest. 116, 1983-1993 (2006).

35. Tsubone, T., Masaki, T., Katsuragi, I., Tanaka, K., Kakuma, T. \& Yoshimatsu, H. Ghrelin regulates adiposity in white adipose tissue and UCP1 mRNA expression in brown adipose tissue in mice. Regul. Pept. 130, 97-103 (2005).

36. Maletinska, L. et al. The Peptidic GHS-R antagonist [D-Lys(3)]GHRP-6 markedly improves adiposity and related metabolic abnormalities in a mouse model of postmenopausal obesity. Mol. Cell Endocrinol. 343, 55-62 (2011).

37. Richard, D. \& Picard, F. Brown fat biology and thermogenesis. Front. Biosci. (Landmark Ed.) 16, 1233-1260 (2011).

38. Yasuda, T., Masaki, T., Kakuma, T. \& Yoshimatsu, H. Centrally administered ghrelin suppresses sympathetic nerve activity in brown adipose tissue of rats. Neurosci. Lett. 349, 75-78 (2003).

39. Choi, K. et al. The role of ghrelin and growth hormone secretagogues receptor on rat adipogenesis. Endocrinology 144, 754-759 (2003).

40. Davies, J.S. et al. Ghrelin induces abdominal obesity via GHS-Rdependent lipid retention. Mol. Endocrinol. 23, 914-924 (2009).

41. Bugge, A., Siersbaek, M., Madsen, M.S., Gondor, A., Rougier, C. \& Mandrup, S. A novel intronic peroxisome proliferator-activated receptor gamma enhancer in the uncoupling protein (UCP) 3 gene as a regulator of both UCP2 and -3 expression in adipocytes. J. Biol. Chem. 285, 17310-17317 (2010).

42. Salans, L.B., Knittle, J.L. \& Hirsch, J. The role of adipose cell size and adipose tissue insulin sensitivity in the carbohydrate intolerance of human obesity. J. Clin. Invest. 47, 153-165 (1968).

43. Kubota, N. et al. PPAR gamma mediates high-fat diet-induced adipocyte hypertrophy and insulin resistance. Mol. Cell 4, 597-609 (1999).

44. Patterson, Z.R., Ducharme, R., Anisman, H. \& Abizaid, A. Altered metabolic and neurochemical responses to chronic unpredictable stressors in ghrelin receptor-deficient mice. Eur. J. Neurosci. 32, 632-639 (2010).

45. Barnett, B.P. et al. Glucose and weight control in mice with a designed ghrelin O-acyltransferase inhibitor. Science 330, 1689-1692 (2010).

46. Toshinai, K. et al. Des-acyl ghrelin induces food intake by a mechanism independent of the growth hormone secretagogue receptor. Endocrinology 147, 2306-2314 (2006).

47. Chen, C.Y. et al. Des-acyl ghrelin acts by CRF type 2 receptors to disrupt fasted stomach motility in conscious rats. Gastroenterology 129, 8-25 (2005).

48. Ariyasu, H. et al. Transgenic mice overexpressing des-acyl ghrelin show small phenotype. Endocrinology 146, 355-364 (2005). 